\title{
OCT/LCT monitoring the drug effect on the human cornea structure in vivo
}

\author{
E.A. Genina ${ }^{1,2 *}$, A.N. Bashkatov ${ }^{1,2}$, I.D. Kamenskikh ${ }^{3}$, I.O. Kolbenev ${ }^{3}$, T.G. Kamenskikh ${ }^{3}$, \\ V.V. Tuchin ${ }^{1,2,4}$ \\ ${ }^{1}$ N.G. Chernyshevsky Saratov State University, 83 Astrakhanskaya Str., Saratov, 410012, Russia \\ ${ }^{2}$ Tomsk State University, 36 Prosp. Lenina, Tomsk, 634050, Russia \\ ${ }^{3}$ V.I. Razumovsky Saratov State Medical University, 112 Bolshaya Kazachya Str., Saratov, 410012, Russia \\ ${ }^{4}$ Institute of Precision Mechanics and Control, Russian Academy of Sciences, 24 Rabochaya Str., Saratov, 410028, \\ Russia \\ *e-mail: eagenina@yandex.ru
}

\begin{abstract}
The effect of anti-glaucoma drugs on the structure components of human eye cornea are studied. The eye drops Timolol-AKOS $0.5 \%$ and Kosopt were used as the object of study. 10 voluntary patients of the Eye Disease Clinic aged from 70 to 75 suffering from glaucoma took part in the studies. The study of cornea in vivo was carried out using the methods of laser scanning confocal tomography (LCT) and optical coherence tomography (OCT) before the application of drugs and at different time moments after the application. The eye cornea thickness values measured using the OCT are presented. From the LCT data the mean diameter of the cornea epithelial cells was calculated. As a result of the complex studies carried out, it was shown that the TimololAKOS medication causes the swelling of the cornea within the limits of $5 \%$. Under the use of Kosopt medication the cornea dehydration was observed. (C) 2015 Samara State Aerospace University (SSAU).
\end{abstract}

Keywords: glaucoma; cornea; optical coherence tomography; confocal microscopy.

Paper \#1989 received 2014.12.17; revised manuscript received 2015.01.21; accepted for publication 2015.01.21; published online 2015.03.28.

\section{References}

1. Y. L. Kim, J. T. Walsh Jr., T. K. Goldstick, and M. R. Glucksberg, "Variation of corneal refractive index with hydration," Phys. Med. Biol. 49, 859-868 (2004).

2. K. V. Larin, and M. G. Ghosn, "Optical coherent tomography measurements of the diffusion rate of water and drugs in an isolated and whole cornea," Quantum Electronics 36(12), 1083-1088 (2006).

3. Y. Wu, D. Clarke, A. Mathew, I. Nicoud, and X. Li, "Noninvasive optical coherence tomography monitoring of structure and hydration changes of human corneas in different preservation media," J. Biomed. Opt. 16(2), 026015 (2011).

4. M. G. Ghosn, V. V. Tuchin, and K. V. Larin, "Nondestructive quantification of analyte diffusion in cornea and sclera using optical coherence tomography," Invest. Ophthalmol. \& Vis. Sci. 48(6), 2726-2733 (2007).

5. M. Bohnke, and B. R. Masters, "Confocal microscopy of the cornea," Progress in Retinal and Eye Research 18(5), 553-628 (1999).

6. S. E. Avetisov, G. B. Egorova, A. A. Fedorov, and N. V. Bobrovskikh, "Confocal microscopy of cornea. Communication 1. The features of normal morphology pattern," Vestnik oftalmologii 3, 3-5 (2008) (in Russian).

7. I. G. Smetankin, and D. I. Agarkova, "Confocal microscopy and optical coherence tomography in the assessment of anatomy and functional condition of a cornea wound (in vivo) after the phacoemulsification of cataract," Sovremennye tekhnologii v medistine 3, 89-92 (2012) (in Russian).

8. Y. Zhou, K. K. H. Chan, T. Lai, and S. Tang, "Characterizing refractive index and thickness of biological tissues using combined multiphoton microscopy and optical coherence tomography," Biomedical Optics Express 4(1), 38-50 (2013). 
9. D. E. Freund, R. L. McCally, and R. A. Farrell, "Effects of fibril orientations on light scattering in the cornea," J. Opt. Soc. Am. A. 3, 1970-1982 (1986).

10. R. A. Farrell, D. E. Freund, and R. L. McCally, "Research on corneal structure," Johns Hopkins Appl. Physics Lab. Techn. Digest. 11(1,2) 191-199 (1990).

11. A. S. Orlova, A. N. Bashkatov, E. A. Genina, I. O. Kolbenev, I. D. Kamenskikh, T. G. Kamenskikh, and V. V. Tuchin, "Influence of 40\%-glucose solution on a human corneal structure," Izvestiya Saratov. Universiteta. New ser. Ser. Physics 14(1), 11-19 (2014).

\section{Introduction}

It is well known that at present glaucoma is one on the main causes of vision loss. The traditional definition of the glaucoma notion incorporates a large group of eye diseases, characterised by permanent or periodic increase of intraocular pressure caused by the disturbed afflux of the aqueous fluid from the eye. The pressure increase results in the development of visual dysfunction, typical for glaucoma, and atrophy with the visual nerve excavation.

The permanent improvement of medicinal preparations and methods of affecting the biological tissue greatly contributes to the struggle for the patients' health, particularly in ophthalmology, and the use of modern optical methods for diagnostics and monitoring the eye disease treatment is now a standard procedure already available in the majority of medical institutions. At the same time, in spite of apparent progress in pharmacology, additional studies of the medication effect on the eye tissue structural elements remain to be an urgent scientific problem, aimed at reducing the possible side effects and increasing the efficiency of the medical treatment procedures.

Optical coherence tomography and confocal microscopy are widely used methods of noninvasive control of the cornea thickness and refractive index, of studying its structural features, and of monitoring the diffusion of drugs into a tissue [1-6]. Combining several visualisation methods allows wider capabilities of diagnosing the structural and optical cornea parameters $[7,8]$.

The goal of the present paper is to study the shortterm effect of anti-glaucoma drugs Timolol-AKOS and Kosopt on the structural components of the human eye cornea at their single topical application.

\section{Human eye cornea structure}

The cornea plays the key role in the optical system of the eye, provided that it is transparent. The cornea has a multilayer structure. From the outer side it is coated with epithelium, consisting of six cellular layers with the thickness of $\sim 40 \mu \mathrm{m}$. The basic cornea substance $(\sim 90 \%)$ consists of transparent connective stroma and squamous cells (keratocytes) occupying $3-5 \%$ of the cornea volume. The stroma thickness amounts to $\sim 500$ $\mu \mathrm{m}$. From the front and from the behind the stroma is adjacent to two terminal plates. The front plate or the Bowman's layer ( $\sim 10 \mu \mathrm{m}$ thick $)$ is a derivative of the corneal base substance. It is separated from the outer epithelium by the $\sim 0.05 \mu \mathrm{m}$ thick membrane. The back plate, or the Descemet's membrane, is a derivative of the endothelium that coats the back surface of the cornea and the anterior chamber of the eye. Together with the endothelium, the thickness of the Descemet's membrane amounts to $\sim 10 \mu \mathrm{m}[9,10]$. Fig. 1 shows sagittal histological section for light microscopy of normal human cornea.

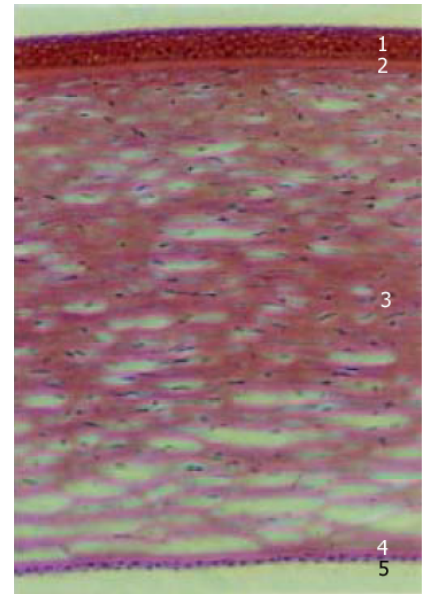

Fig. 1 Sagittal histological section for light microscopy of normal human cornea: 1 is the epithelium layer; 2 is the Bowman's layer; 3 is the stroma; 4 is the Descemet's membrane; 5 is the endothelium layer. Total corneal thickness is $540 \mu \mathrm{m}[5]$.

\section{Materials and methods of the study}

The study was carried out in the Clinic of Eye Diseases, V.I. Razumovsky Saratov State Medical University.

The eye drops Timolol-AKOS 0.5\% (Sintez, Russia) and Kosopt (MSD, USA and Canada) were used as the studied drugs.

The eye drops Timolol-AKOS $0.5 \%$ ( $1 \mathrm{ml}$ contains $6.8 \mathrm{mg}$ of the active substance timolol maleate and adjuvants, namely, disodium phosphate dodecahydrate, sodium dihydrophosphate monohydrate, disodium edetate dihydrate, sodium chloride, benzalconium chloride, sodium hydroxide, purified water) are an antiglaucoma drug, a nonselective beta-adrenoblocker. Being topically applied, Timolol reduces the intraocular pressure at the expense of decreasing the aqueous fluid formation and minor increase of its drainage. The $\mathrm{pH}$ value of the preparation measured using the universal indicator paper (Likont, Perm) amounted to $\sim 7$.

The eye drops Kosopt $(1 \mathrm{ml}$ contains the active substances, $22.26 \mathrm{mg}$ of dorzolamide hydrochloride and 
$6.83 \mathrm{mg}$ of timolol maleate, and the adjuvants, namely, benzalconium chloride, sodium citrate, mannitol, hydroxyethyl cellulose, sodium hydroxide, water for injections) are used to treat the increased intraocular pressure in the cases of open-angle glaucoma and pseudoexfoliative glaucoma. The combined action of active substances leads to more expressed reduction of intraocular pressure at the expense of reducing the secretion of intraocular fluid, probably due to the decreased production of bicarbonate ions, which, in turn, causes the deceleration of sodium and fluid transport. The $\mathrm{pH}$ value of the preparation amounts to $\sim 5$.

The refractive index values of the medicinal preparations under study, measured using the Abbe refractometer at the wavelength $589 \mathrm{~nm}$, were close to the refractive index of saline $(0.09 \% \mathrm{NaCl}) \sim 1.335$.

The study of cornea in vivo was carried by means of laser scanning confocal tomography (LCT) using the confocal tomograph Heidelberg Retina Tomograph (HRT-II) with the cornea module Rostok Cornea Module (RCM) (Heidelberg Engineering $\mathrm{GmbH}$, Germany) that allowed layer-specific imaging of the cornea, limb and conjunctive, as well as the epithelium cells counting and intracorneal pachymetry. Optical coherence tomography (OCT) was carried out using the OCT tomograph Topcon 3D OCT-1000 (Topcon, Japan).

The light source used in the LCT system was a diode laser with the wavelength $670 \mathrm{~nm}$. The studied area was $400 \times 400 \mu \mathrm{m}$, the image size was $384 \times 384$ pixels, the axial resolution was $4 \mu \mathrm{m}$ and the lateral one was $2 \mu \mathrm{m}$. The image recording rate was 30 frames per second. The examination procedure included the topical anaesthesia and the exposure of the contact gel that was applied to the water immersion objective. We used the manual examination regime, and the measurement of cell diameters was semiautomatic. Fig. 2 presents the image of the human eye corneal epithelium, obtained using the confocal retinal tomograph.

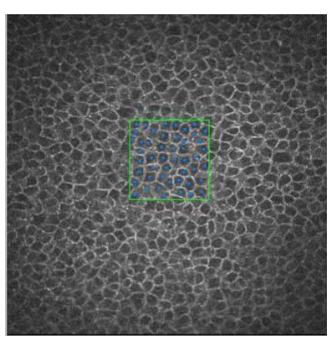

Fig. 2 Image of human eye cornea epithelium obtained by means of confocal microscopy. In the image centre the area in which the cells are counted is selected.

In the OCT tomograph the light source was a superluminescent diode with the wavelength $840 \mathrm{~nm}$. The optical power at the cornea surface was $650 \mu \mathrm{W}$ with the frequency $6.5 \mathrm{~Hz}$, the axial resolution $6 \mu \mathrm{m}$, and the lateral resolution $20 \mu \mathrm{m}$.

Ten volunteers of the Clinic of Eye Diseases aged from 70 to 75 suffering from glaucoma took part in the investigations. The patients were randomly divided into two groups 5 persons in each. One of the groups was treated with Timolol-AKOS and the other one with Kosopt. The drugs were dropped into both eyes.

The OCT/LCT studies were performed before the application of the drugs and in 30-40 minutes after the application. After each measurement the mean diameter of the cornea epithelial cells were calculated from the LCT data. (Fig. 2). From the OCT data the mean cornea thickness was calculated for every group (Fig. 3). The mean diameter of the cornea epithelial cells and the mean cornea thickness were measured with built-in software of the used devices.

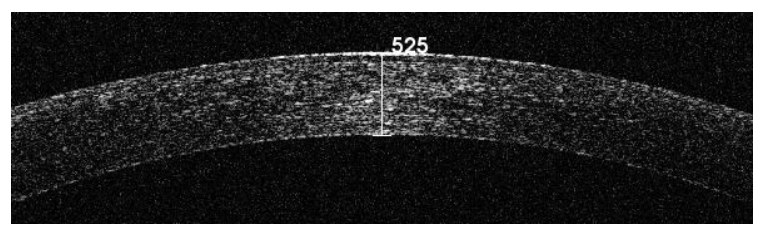

Fig. 3 Image of the human eye cornea cross section obtained by means of the optical coherence tomography. The figures indicate the cornea thickness in $\mu \mathrm{m}$.

The comparison was carried out using the Student's test between the initial value of the studied parameter averaged over the group and its posterior values at the moment of registration also averaged over the group. The differences were considered to be statistically significant in the case when the value of the parameter $p$ was smaller than 0.05 .

\section{Results and discussion}

Fig. 4 shows the values of the cornea thickness at the initial moment and at different periods after the application of the studied preparations. It is well seen that Timolol-AKOS causes short-time swelling of the tissue; the cornea thickness has first grown by $3 \%$ upon the average during 20 minutes of observation and then began to decrease. Kosopt causes the reduction of the cornea thickness by $5 \%$ upon the average during 30 minutes of observation.

Fig. 5 presents the effect of the studied drugs on the size of cornea epithelium cells. Both Timolol-AKOS and Kosopt increase the cell diameter by 5\% (in $40 \mathrm{~min}$ ) and $1 \%$ (in $30 \mathrm{~min}$ ), respectively.

The statistical analysis of the studied parameters has shown that the increase of the mean cornea thickness in 20 minutes after the application of Timolol-AKOS in comparison with the initial values is statistically significant. The increase of the epithelial cells diameter during the same period of time is statistically significant as well.

The comparison of the mean cornea thickness at the initial moment of time and in 30 minutes after the application of Kosopt has demonstrated statistical significance of the difference. However, the variations of the epithelial cell diameter appeared to be insignificant, so that it is possible to conclude that this 
preparation does not essentially affect the cornea epithelial cells.

From the experimental data presented above it is seen that the anti-glaucoma preparations TimololAKOS and Kosopt, executing their main function of reducing the intraocular pressure by decreasing the amount of the produced intraocular fluid, affect the eye cornea in a slightly different way. Thus, Timolol-AKOS causes the cornea swelling, while after the use of Kosopt the cornea thickness decreases. Besides the different composition of the drugs (side effect of timolol maleate is edema of the corneal epithelium), this effect may be explained by the different $\mathrm{pH}$ values of the solutions. The experiments with the rabbit cornea in vitro described earlier in Ref. [11] demonstrated that the hydration degree of the cornea decreases and reaches its minimum in 20 minutes after putting the tissue sample into the buffer solution with $\mathrm{pH}=5$. From Fig. 4 it is seen that Kosopt having $\mathrm{pH} \sim 5$, causes the reduction of the cornea thickness, while Timolol-AKOS having $\mathrm{pH}$ $\sim 7$ causes the tissue swelling.

\section{Conclusion}

As a result of the performed complex studies of the effect of anti-glaucoma drugs on the condition of the cornea using the optical coherence tomography and laser confocal tomography it was shown that the preparation Timolol-AKOS $0.5 \%$ causes the cornea swelling within the limits of $5 \%$, which also affects the increase of the epithelial cells diameter. On the contrary, the use of Kosopt leads to the observable cornea dehydration.

The work was carried out within the frameworks of the Programme for Increasing the Competitive Ability of Tomsk State University and under the partial support from the President of Russia, grant "Leading Scientific Schools" No. 1177.2012.2.
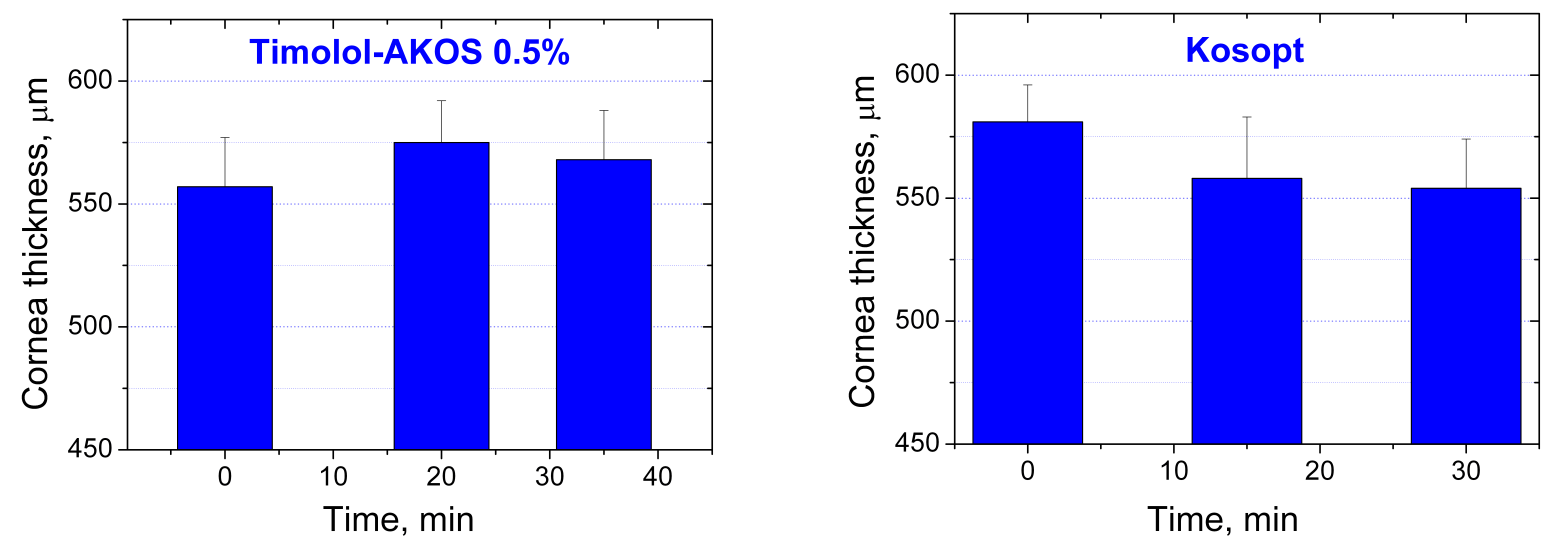

Fig. 4 Human eye cornea thickness in vivo at different time moments under the action of the studied drugs.
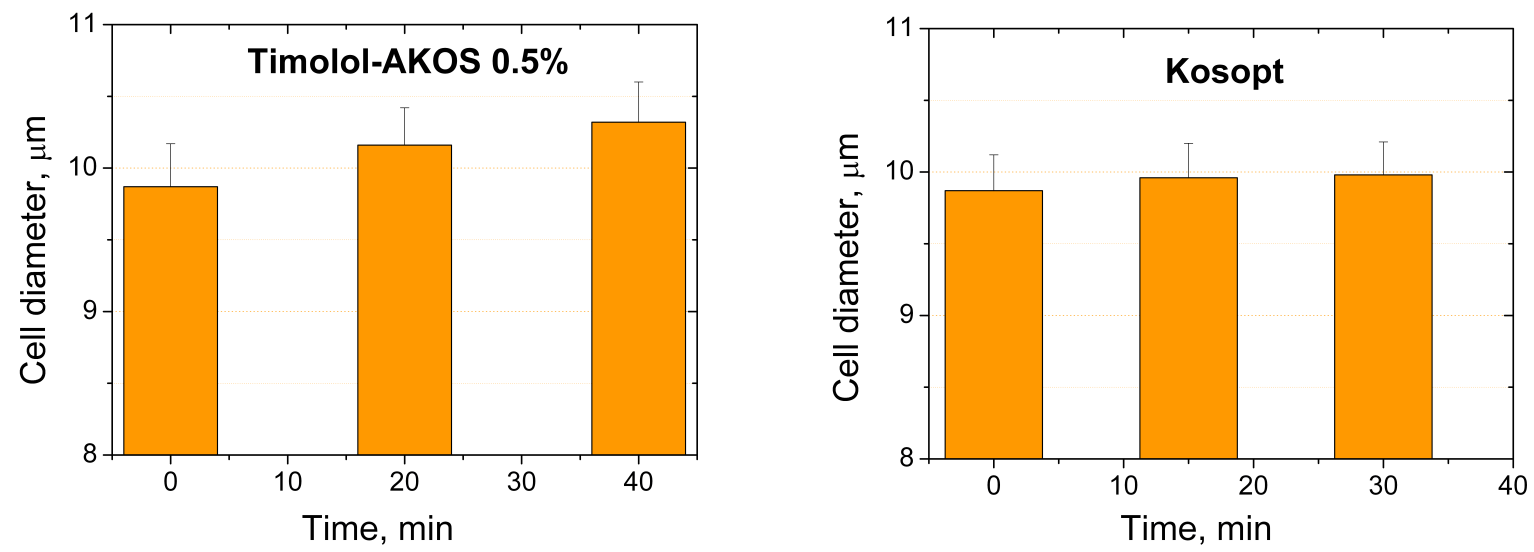

Fig. 5 Human eye cornea epithelium cell diameter in vivo in different periods of the action of the studied drugs. 